\title{
EXTRAGALACTIC 1 MILLIMETER SOURCES: SIMULTANEOUS OBSERVATIONS AT CENTIMETER, MILLIMETER, AND VISUAL WAVELENGTHS
}

\author{
R. LANDAU AND T. W. JONES \\ Astronomy Department, University of Minnesota \\ E. E. EPSTEIN \\ Electronics Research Laboratory, The Aerospace Corporation \\ G. Neugebauer and B. T. Solfer \\ Palomar Observatory, California Institute of Technology \\ M. W. WERNER \\ NASA Ames Research Center \\ J. J. PUSCHELL \\ Center for Astrophysics and Space Science, University of California, San Diego \\ AND \\ T. J. BALONEK \\ Department of Physics and Astronomy, University of Massachusetts \\ Received 1982 September 30; accepted 1982 October 27
}

\begin{abstract}
Observations simultaneously made at visual $(B, V$, and $R$ ) wavelengths, at $1,1.4$, and $3.4 \mathrm{~mm}$ and at 1.3, 2, 6, and $20 \mathrm{~cm}$ of 9 QSOs and BL Lac objects are reported. The range of the millimetervisual spectral index $\alpha_{\mathrm{mv}}$ was only $0.65-0.82$, typical of optically thin synchrotron emission. This may indicate that the electrons radiating synchrotron emission in this portion of the spectrum are not subjected to large radiative losses, and therefore relativistic bulk motion with Doppler factors $\sim 10$ are required. The visual spectral index is much more broadly distributed and typically larger than $\alpha_{\mathrm{mv}}$. The spectral energy distributions have not changed much in the last 2-5 years except for $2251+15$ and perhaps $0235+164$. Only $1749+09$ shows a sharp spectral break shortward of $1 \mathrm{~mm}$. Sixteen other sources were observed at $1 \mathrm{~mm}$, of which seven were detected.
\end{abstract}

Subject headings: BL Lacertae objects - infrared: sources - quasars - radiation mechanisms radio sources: general

\section{INTRODUCTION}

Studies of the spectra of compact nonthermal extragalactic radio sources have been plagued by the sparsity of observations between $3 \mathrm{~mm}$ and $10 \mu \mathrm{m}$ and by the variability of the sources which makes a spectrum difficult to define unless observations at all frequencies are made essentially simultaneously. Observations at $1 \mathrm{~mm}$ and at submillimeter wavelengths are crucial because the transition from flat, optically thick radio emission to the weaker, steep-spectrum, visual-infrared radiation occurs typically in this region. The nature of this transition - whether abrupt or gradual, and at what frequency it occurs - can be used to restrict models of the structure and energetics of these sources. Submillimeter or $1-\mathrm{mm}$ observations of nonthermal extragalactic sources have been made by Hildebrand et al. (1977), Elias et al. (1978), Jones et al. (1981), and Ennis, Neugebauer, and Werner (1982). Jones et al. report simultaneous radio observations for several sources. We present here observations at $1 \mathrm{~mm}$ and simultaneous observations at both longer and shorter wavelengths.

The need for simultaneous observations to define the spectrum arises from the rapid variability of many of these sources. The minimum time scale of variability, closely related to the size of the source components, is generally a function of wavelength. At optical wavelengths, time scales are often days, although more rapid variations are seen in several sources. The best studied of these are BL Lac (Racine 1970), OJ 287 (Kinman et al. 1974), and AO 0235+164 (Rieke et al. 1976). In the radio domain, time scales are typically weeks or longer, although again, more rapid events have been observed many times (see the discussion in the appendix of Epstein, Landau, and Rather 1980).

The observations presented here were all made within the 10 day period 1981 December 5-15. It was our intention to cover the wavelength range from $4000 \AA$ to $20 \mathrm{~cm}$ as completely as possible. Measurements were obtained with a broad-band filter photometer in the visual at $B, V$, and $R$; with a broad-band detector centered at $1.0 \mathrm{~mm}$; with coherent receivers at $1.4 \mathrm{~mm}$ and $3.4 \mathrm{~mm}$; and at the four VLA wavelengths: $1.3,2$, 6 , and $20 \mathrm{~cm}$. Planned observations in the infrared at 2 , 10 , and $20 \mu \mathrm{m}$ and in the submillimeter region were prevented by inclement weather. It was our further intention to study a sufficiently large number of sources 
TABLE 1

JOURNAL OF OBSERVATIONS, 1981 DECEMBER

\begin{tabular}{|c|c|c|c|c|c|c|c|c|c|c|c|c|}
\hline \multirow[b]{2}{*}{ TELESCOPE } & \multirow[b]{2}{*}{ WAVELENGTH(s) } & \multicolumn{11}{|c|}{ DATE (UT) } \\
\hline & & 5 & 6 & 7 & 8 & 9 & 10 & 11 & 12 & 13 & 14 & 15 \\
\hline VLA & $20,6,2,1.3 \mathrm{~cm}$ & & & & & $x$ & & & & & $x$ & \\
\hline FCRAO $^{\mathrm{a}} 13.7 \mathrm{~m} \ldots \ldots \ldots$ & $3.4 \mathrm{~mm}$ & $\times$ & & & & & & & & & & \\
\hline NRAO $^{\mathrm{b}} 11 \mathrm{~m} \ldots \ldots \ldots \ldots$ & $1.4 \mathrm{~mm}$ & & & & $x$ & $\times$ & $x$ & $\times$ & & & & \\
\hline Hale $5 \mathrm{~m} \ldots \ldots \ldots \ldots \ldots \ldots$ & $1.0 \mathrm{~mm}$ & & & & & & & & & & $x$ & \\
\hline UM-UCSD $1.5 \mathrm{~m} \ldots \ldots \ldots$ & $B, V, R$ & & & & & & & & & $x$ & $x$ & $x$ \\
\hline
\end{tabular}

a The Five College Radio Astronomy Observatory is operated with support from the National Science Foundation under grant AST-80-26702 and with permission of the Metropolitan District Commision, Commonwealth of Massachusetts.

b The National Radio Astronomy Observatory is operated by Associated Universities, Inc., under contract with the National Science Foundation.

to assess the representativeness of those that have been well studied. With our sample of nine sources that have nearly complete wavelength coverage we are now able to define common features and to note exceptions.

\section{OBSERVATIONS}

The division of labor and dates of observations are given in Table 1. Table 2 lists the 25 sources observed at $1.4 \mathrm{~mm}$ with their formal $1 \sigma$ uncertainties. Table 3 summarizes the observations of the 15 sources that were observed at more than one wavelength. Except for having flat radio spectra, the sources were not chosen in any systematic way. They are, in fact, largely BL Lac objects and highly polarized quasars. Details of the observing procedures, calibration, and data reduction at the five telescopes are given below.

\section{a) $1.4 \mathrm{~mm}$ Observations}

Observations at the $11 \mathrm{~m}$ telescope were made with a cooled heterodyne receiver operating at $226 \mathrm{GHz}$ with a noise temperature of $800 \mathrm{~K}$. The bandwidth was $800 \mathrm{MHz}$. "On-on" beam-switching was used with a throw of $5^{\prime}$ in azimuth, a chopping rate of $83 \mathrm{~Hz}$, and a beam-switching rate of about 1 per minute. Pointing was calibrated by frequent positional observations of the flux calibrators Venus, Mars, Jupiter, and Saturn. Extinction was monitored both by making "tipping" scans and by measuring the water vapor content of the atmosphere in the line of sight to the Moon or the Sun with a spectral hygrometer (Landau 1982). For the first $80 \%$ of the observing period, the zenith optical depth was 0.14 \pm 0.04 ; it then rose to $0.35 \pm 0.02$ as weather conditions deteriorated. At the same time the gain of the system gradually decreased by $30 \%$ as inferred from a decrease in the measured flux of the calibrators. Both these effects were taken into account in reducing the data.

Absolute fluxes were determined by assuming the following brightness temperatures for the calibrators: Venus, $290 \mathrm{~K}$; Mars, $215 \mathrm{~K}$; Jupiter, $155 \mathrm{~K}$; Saturn, $140 \mathrm{~K}$ (based on measurements at longer wavelengths by Ulich 1981 and at shorter wavelengths by Whitcomb et al. 1979). To set the focus, the flux as a function of focus position was measured at 19 positions over $60 \mathrm{~mm}$. These focus curves had a reproducible shape with two maxima but showed a slight shift in positions between day and night. The focus was always set to one of the peaks. For each source $n 5$ minute integrations were made, each consisting of many off-on-on-off sequences. Table 2 lists the values of $n$. The errors quoted in Tables 2 and 3 are the standard errors of the mean of the $n$ integrations. The

TABLE 2

$226 \mathrm{GHz}(1.4 \mathrm{~mm})$ Flux Densities (Jy)

\begin{tabular}{|c|c|c|c|c|c|}
\hline Source & $S_{226} \pm \sigma$ & $n^{\mathrm{a}}$ & Source & $S_{226} \pm \sigma$ & $n^{\mathrm{a}}$ \\
\hline $0007+10$, III Zw 2 & $-0.6 \pm 0.3$ & 6 & $1226+02,3 \mathrm{C} 273$. & $9.4 \pm 0.4$ & 14 \\
\hline $0109+22 \ldots \ldots \ldots \ldots$ & $0.3 \pm 0.9$ & 6 & $1308+32 \ldots \ldots \ldots$ & $1.5 \pm 0.6$ & 6 \\
\hline $0235+164 \ldots \ldots \ldots \ldots \ldots \ldots$ & $3.3 \pm 0.3$ & 15 & $1418+54$ & $1.1 \pm 1.1$ & 6 \\
\hline $0316+413,3 \mathrm{C} 84 \ldots \ldots \ldots \ldots$ & $27.2 \pm 0.8$ & 5 & $1633+38 \ldots \ldots \ldots \ldots$ & $-0.2 \pm 0.7$ & 6 \\
\hline $0355+50,4 C 50.11 \ldots \ldots \ldots \ldots$ & $3.2 \pm 0.4$ & 25 & $1641+39,3 \mathrm{C} 345 \ldots \ldots \ldots \ldots$ & $9.9 \pm 1.0$ & 11 \\
\hline $0415+37,3 \mathrm{C} 111 \ldots \ldots \ldots \ldots$ & $1.5 \pm 0.4$ & 17 & $1727+50 \ldots \ldots \ldots \ldots \ldots \ldots$ & $0.1 \pm 0.2$ & 4 \\
\hline $0422+00 \ldots \ldots \ldots \ldots \ldots \ldots \ldots$ & $1.1 \pm 0.3$ & 20 & $1749+09 \ldots \ldots \ldots \ldots$ & $0.9 \pm 0.5$ & 17 \\
\hline $0430+05,3 C 120 \ldots \ldots \ldots \ldots$ & $1.7 \pm 0.5$ & 20 & $1807+698,3$ C $371 \ldots$ & $1.0 \pm 0.9$ & 9 \\
\hline $0735+178 \ldots \ldots \ldots \ldots \ldots \ldots$ & $1.1 \pm 0.4$ & 18 & $2134+00 \ldots \ldots \ldots$ & $2.0 \pm 0.9$ & 3 \\
\hline $0736+017 \ldots \ldots \ldots \ldots \ldots \ldots$ & $1.2 \pm 0.5$ & 16 & $2200+42$, BL Lac $\ldots \ldots \ldots \ldots$ & $2.0 \pm 0.5$ & 25 \\
\hline $0851+202$, OJ $287 \ldots$ & $4.2 \pm 0.4$ & 18 & $2223-05,3 \mathrm{C} 446 \ldots \ldots \ldots \ldots$ & $3.5 \pm 0.7$ & 18 \\
\hline $1101+384, \mathrm{Mk} 421 \ldots$ & $0.6 \pm 0.6$ & 10 & $2251+15,3 \mathrm{C} 454.3 \ldots$ & $4.1 \pm 0.7$ & 12 \\
\hline $1219+29$, W Com $\ldots \ldots \ldots \ldots$ & $1.4 \pm 0.8$ & 8 & & & \\
\hline
\end{tabular}

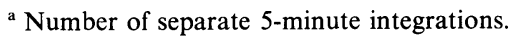


TABLE 3

Multifrequency Flux Densities

\begin{tabular}{|c|c|c|c|c|c|c|c|c|c|c|}
\hline $\begin{array}{l}\text { Wavelength: } \\
\text { Frequency }(\mathrm{Hz}) \text { : }\end{array}$ & $\begin{array}{c}20 \mathrm{~cm} \\
1.5(9) \\
(\mathrm{Jy})\end{array}$ & $\begin{array}{c}6 \mathrm{~cm} \\
4.9(9) \\
(\mathrm{Jy})\end{array}$ & $\begin{array}{c}2 \mathrm{~cm} \\
1.5(10) \\
(\mathrm{Jy})\end{array}$ & $\begin{array}{c}1.3 \mathrm{~cm} \\
2.25(10) \\
(\mathrm{Jy})\end{array}$ & $\begin{array}{c}3.4 \mathrm{~mm} \\
8.7(10) \\
(\mathrm{Jy})\end{array}$ & $\begin{array}{c}1.4 \mathrm{~mm} \\
2.26(11) \\
(\mathrm{Jy})\end{array}$ & $\begin{array}{c}1.0 \mathrm{~mm} \\
3.0(11) \\
(\mathrm{Jy})\end{array}$ & $\begin{array}{c}0.70 \mu \mathrm{m} \\
4.3(14) \\
(\mathrm{mJy})\end{array}$ & $\begin{array}{c}0.55 \mu \mathrm{m} \\
5.5(14) \\
(\mathrm{mJy})\end{array}$ & $\begin{array}{c}0.44 \mu \mathrm{m} \\
7.0(14) \\
(\mathrm{mJy})\end{array}$ \\
\hline $0235+164$ & 1.32 & 2.21 & 3.28 & 3.20 & $4.0 \pm 0.2$ & $3.3 \pm 0.3$ & $4.5 \pm 1.0^{\mathrm{a}}$ & $15.0 \pm 2.0$ & $6.6 \pm 1.0$ & $3.8 \pm 0.6$ \\
\hline $0355+50,4 C 50.11$ & $5.45^{\mathrm{b}}$ & $8.46^{\mathrm{b}}$ & 7.40 & 7.20 & $5.5 \pm 0.2$ & $3.2 \pm 0.4$ & $3.8 \pm 0.9^{\mathrm{a}}$ & $0.8 \pm 1.3^{\mathrm{c}}$ & $1.7 \pm 3.0$ & $\leq \overline{5.0}$ \\
\hline $0415+37,3 C 111 \ldots$ & $\ldots$ & $1.60^{\mathrm{b}}$ & 1.41 & 1.36 & $\ldots$ & $1.5 \pm 0.4$ & $1.1 \pm 0.5^{\mathrm{a}}$ & $\ldots$ & $\ldots$ & $\ldots$ \\
\hline $0422+00 \ldots \ldots \ldots$ & $0.84^{\mathrm{b}}$ & 1.35 & 1.25 & 1.10 & $\ldots$ & $1.1 \pm 0.3$ & $\ldots$ & $\ldots$ & $\ldots$ & $\ldots$ \\
\hline $0430+05,3 \mathrm{C} 120$ & $3.54^{\mathrm{b}}$ & $3.50^{\mathrm{b}}$ & 2.62 & 2.20 & $2.0 \pm 0.3$ & $1.7 \pm 0.5$ & $3.9 \pm 1.0^{\mathrm{a}}$ & $8.5 \pm 1.1$ & $5.3 \pm 0.7$ & $3.0 \pm 0.4$ \\
\hline $0735+178 \ldots \ldots \ldots$ & 2.06 & 2.01 & 1.85 & 1.55 & $1.4 \pm 0.2$ & $1.1 \pm 0.4$ & $\ldots$ & $8.0 \pm 0.9$ & $6.4 \pm 0.7$ & $5.5 \pm 0.6$ \\
\hline $0736+017 \ldots \ldots$ & 2.18 & 2.30 & 2.85 & 2.30 & $1.7 \pm 0.2$ & $1.2 \pm 0.5$ & $\ldots$ & $2.5 \pm 0.4$ & $1.9 \pm 0.4$ & $1.0 \pm 0.6$ \\
\hline $0851+202$, OJ 287 & 1.87 & 3.60 & 6.00 & 5.40 & $6.8 \pm 0.2$ & $4.2 \pm 0.4$ & $5.0 \pm 0.9^{\mathrm{a}}$ & $9.8 \pm 0.6$ & $7.0 \pm 0.5$ & $5.4 \pm 0.3$ \\
\hline $1101+384, \mathrm{Mk} 421 \ldots \ldots \ldots \ldots \ldots$ & $\ldots$ & $\ldots$ & $\ldots$ & $\ldots$ & $\ldots$ & $0.6 \pm 0.6$ & $0.6 \pm 0.6^{\mathrm{a}}$ & $34.0 \pm 2.0$ & $26.0 \pm 2.0$ & $21.0 \pm 1.0$ \\
\hline $1226+02,3 \mathrm{C} 273 \ldots \ldots \ldots \ldots \ldots$ & $32.0^{\mathrm{d}}$ & $26.7^{\mathrm{e}}$ & 33.0 & $\ldots$ & $19.6 \pm 0.4$ & $9.4 \pm 0.4$ & $10.5 \pm 1.6$ & $30.0 \pm 2.0$ & $28.0 \pm 2.0$ & $26.0 \pm 2.0$ \\
\hline $1641+39,3 \mathrm{C} 345 \ldots \ldots \ldots \ldots \ldots$ & $\ldots$ & $\ldots$ & $\ldots$ & $\cdots$ & $13.0 \pm 0.6$ & $9.9 \pm 1.0$ & $10.8 \pm 1.3$ & $\ldots$ & $\ldots$ & $\ldots$ \\
\hline $1749+09 \ldots \ldots \ldots \ldots \ldots \ldots \ldots \ldots$ & 1.37 & 1.91 & 2.36 & 3.20 & $1.9 \pm 0.3$ & $0.9 \pm 0.5$ & $\ldots$ & $\ldots$ & $\ldots$ & $\ldots$ \\
\hline $2200+42$, BL Lac $\ldots$ & 4.60 & 5.70 & 5.10 & 5.40 & $3.2 \pm 0.2$ & $2.0 \pm 0.5$ & $2.9 \pm 0.8$ & $14.0 \pm 8.0$ & $10.0 \pm 6.0$ & $7.0 \pm 4.0$ \\
\hline $2223-05,3 \mathrm{C} 446$ & $5.60^{\mathrm{b}}$ & 4.03 & 4.90 & 6.30 & $5.1 \pm 0.3$ & $3.5 \pm 0.7$ & $3.4 \pm 0.8$ & $1.2 \pm 0.3$ & $1.0 \pm 0.2$ & $0.9 \pm 0.3$ \\
\hline
\end{tabular}

a Observations made 1981 October 12, two months before those in the rest of the table.

${ }^{b}$ Visibility plots indicate the source is being resolved. The total flux is given.

c A true detection. The large error is due to the uncertainty in the correction for galactic absorption. The uncorrected flux density is $0.12 \pm 0.04 \mathrm{mJy}$.

d Measurements on 1981 February 15 and 1982 March 21 both give $32.0 \mathrm{Jy}$.

e Interpolation between 23.0 Jy measured on 1980 November 4 and 27.6 Jy measured 1982 March 21.

limiting accuracy of a set of observations is determined by systematic errors, presumably "hot" and "cold" spots in the dome through which part of the beam moves as it tracks a source. Averaging this source of noise takes many 5 minute integrations. An additional uncertainty of $10 \%$ should be added for errors in the absolute flux calibration.

\section{b) $1.0 \mathrm{~mm}$ Observations}

Observations at the $5 \mathrm{~m}$ telescope were made with a liquid ${ }^{3} \mathrm{He}$-cooled germanium/sapphire composite bolometer (Roellig 1980). The bandwidth of approximately $0.5 \mathrm{~mm}$ was set by filters at the short-wavelength end and limited by diffraction at the telescope aperture at the long-wavelength end. The exact spectral response is determined by the atmospheric water vapor content. Measurements with a spectral hygrometer (Westphal 1974), made just before these observations, showed $3 \mathrm{~mm}$ of precipitable $\mathrm{H}_{2} \mathrm{O}$ airmass ${ }^{-1}$, implying an effective wavelength of $0.9 \mathrm{~mm}$. Flux densities of all sources were referred to a wavelength of $1.0 \mathrm{~mm}$ by assuming their spectra were power laws $\left(S \propto v^{-\alpha}\right)$ with an index of zero, typical of compact sources at this frequency. An error of 0.5 in the spectral index results in no more than a $10 \%$ error in the flux density. Further details of the observing procedures can be found in Elias et al. (1978).

Absolute fluxes were determined by comparison with observations of Venus and Jupiter, assumed to have brightness temperatures of $276 \pm 14 \mathrm{~K}$ and $168 \pm 8 \mathrm{~K}$, respectively (Werner et al. 1978). (Observations in 1981 October shown in Table 3 were calibrated with respect to Mars, assumed to have $\left\langle T_{B}\right\rangle=200 \mathrm{~K}$.) A comparison of the five sources from Table 3 observed simultaneously at both 1.0 and $1.4 \mathrm{~mm}$ shows good agreement within the errors. Furthermore, observational errors at these wave- lengths are still sufficiently large that measurements are not calibration limited; the differences in the adopted brightness temperatures of the calibrators make no difference in the interpretation of the measurements.

\section{c) $1.3,2,6$, and $20 \mathrm{~cm}$ Observations}

At the VLA, each source was observed once in the C configuration for $6,5,3$, and 3 minutes at 1.3, 2, 6, and $20 \mathrm{~cm}$, respectively. The object $3 \mathrm{C} 48$ was the calibrator with assumed flux densities of $1.09,1.75,5.56$, and $15.37 \mathrm{Jy}$, respectively. Total fluxes were estimated from the visibility plots. Where there was evidence that the source was being resolved, a note appears in Table 3 . The baselines used were not long enough to determine the flux of the point source. In all cases the signal-to-noise ratio of the measurement exceeded 100 so no errors are given in Table 3; an uncertainty in the calibration of $10 \%$ at 1.3 and $2 \mathrm{~cm}$ and of $5 \%$ at 6 and $20 \mathrm{~cm}$ should be assumed.

\section{d) $3.4 \mathrm{~mm}$ Observations}

Observations at the Five College Radio Astronomy Observatory were made with a cooled heterodyne receiver operating at $87.3 \mathrm{GHz}$ with a $1.4 \mathrm{GHz} \mathrm{IF}$ of $400 \mathrm{MHz}$ bandwidth. The system noise temperature was $350 \mathrm{~K}$, double sideband; the receiver temperature was $65 \mathrm{~K}$. Chopping was done in azimuth at $11 \mathrm{~Hz}$ with a throw of $6^{\prime}$ and with $8 \mathrm{~s}$ integration times between beam switches. Observations were made sequentially in the two orthogonal linear polarizations and summed. Calibration was with respect to Jupiter with an assumed brightness temperature of $180 \mathrm{~K}$ (seven observations); Saturn, $150 \mathrm{~K}$ (three observations); Venus, $360 \mathrm{~K}$ (three observations); and DR 21, $16.9 \mathrm{~K}$ (three observations). The quoted error is the quadrature sum of the measurement 
and calibration errors. Further details of the observing and reduction procedures may be found in Balonek (1982). The zenith optical depth during the observations was $\tau=0.25$.

$$
\text { e) } 0.44 \mu \mathrm{m}(B), 0.55 \mu \mathrm{m}(V) \text {, and } 0.70 \mu \mathrm{m}(R)
$$

Observations at Mount Lemmon were made with a GaAs photomultiplier through an $18^{\prime \prime}$ aperture using filters described by O'Dell et al. (1978). Landolt (1973) standard stars with $R$ photometry by Kunkel and Rydgren (1979) were used to transform to the Johnston standard system. Mean extinction coefficients for the observatory were used, and the residuals of the transformation were $0.02 \mathrm{mag}$. Standards were observed before and after each source, and most sources were measured twice. Two corrections for galactic absorption were computed; one by the method of Sharov (1964), and one by that of Sandage (1972). An average of the two was used to correct the fluxes to those that would be received outside our Galaxy, and the discordance of the two provided an estimate of the uncertainty in the correction. For sources at low galactic latitude, especially BL Lac and $0355+50(4 \mathrm{C} 50.11)$, this uncertainty dominates the error in the flux density; for $3 \mathrm{C} 120,0735+178$, and $0235+164$ it is about $12 \%$; for all other sources, about $5 \%$. Extinctions calculated from the data of Burstein and Heiles (1982) generally agree within the errors with those derived above. We retain the latter for comparison (below) with measurements at previous epochs which were similarly reduced. The errors given in Table 3 are the combined errors in the photometry, in the transformation, and in the correction for galactic absorption. The conversion from magnitude to absolute flux density is given in O'Dell et al. (1978); it is assumed to be accurate to $5 \%$.

In Table 4 we list two spectral indices: $\alpha_{\mathrm{mv}}$ between $1.4 \mathrm{~mm}$ and $R$, and $\alpha_{\text {vis }}$ between $R$ and $B$ for those sources with accurate photometry at these wavelengths. The errors in $\alpha_{\mathrm{mv}}$ come from propagating the errors in the flux densities from Table 3. The index $\alpha_{\text {vis }}$ is the slope of the regression line through the $B, V$, and $R$ points. Its error is the larger of the error in the slope determined from the regression or the propagation of the photometric error in the flux densities. The uncertainty in the correction for galactic extinction does not affect the spectral

\begin{tabular}{|c|c|c|}
\hline Source & $\alpha_{\mathrm{mv}}$ & $\alpha_{\text {vis }}$ \\
\hline $0235+164$ & $0.71 \pm 0.03$ & $2.82 \pm 0.30$ \\
\hline $0430+05,3 C 120$ & $0.70 \pm 0.06$ & $2.14 \pm 0.13$ \\
\hline $0735+178 \ldots \ldots \ldots$ & $0.65 \pm 0.07$ & $0.77 \pm 0.10$ \\
\hline $0736+017 \ldots \ldots \ldots$ & $0.82 \pm 0.08$ & $1.88 \pm 0.45$ \\
\hline $0851+202$, OJ 287 & $0.80 \pm 0.02$ & $1.22 \pm 0.10$ \\
\hline $1226+02,3 C 273$ & $0.76 \pm 0.02$ & $0.29 \pm 0.04$ \\
\hline $2200+42$, BL Lac. & $0.66 \pm 0.12$ & $1.42 \pm 0.06$ \\
\hline $2223-05,3 \mathrm{C} 446$ & $1.06 \pm 0.06$ & $0.59 \pm 0.09$ \\
\hline
\end{tabular}

TABLE 4 index except through our uncertainty in the slope of the interstellar extinction curve.

\section{DISCUSSION}

The list of Table 2 increases by nine the number of nonthermal extragalactic sources observed at $1 \mathrm{~mm}$ and

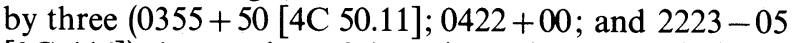
[3C 446]) the number of detections. An upper limit for $1749+09$ is also useful in conjunction with the radio measurements. Including those sources reported by Elias et al. (1978), Jones et al. (1981), and Ennis, Neugebauer, and Werner (1982), the number of such $1 \mathrm{~mm}$ detections is now 30. We discuss first the spectra defined by our measurements and then their variations since 1977 January.

\section{a) The Spectra}

The radio/1 $\mathrm{mm} /$ visual spectra of nine sources are

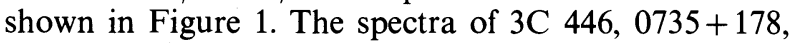
and $4 \mathrm{C} 50.11$ are shown here for the first time. In some respects the spectra are qualitatively similar to one another. For example the mean spectral index, $\alpha\left(S \sim v^{-\alpha}\right)$, between $1.4 \mathrm{~mm}$ and $0.70 \mu \mathrm{m}$ for the sources of Table 4 (but exclusive of $3 \mathrm{C} 446$ ) is $\left\langle\alpha_{\mathrm{mv}}\right\rangle=0.73$ with a sample standard deviation of only 0.07 . (3C 446 appears to have $\alpha_{\mathrm{mv}}=1.06 \pm 0.06$ and may deserve further study.)

The uniformity of the millimeter-to-visual spectral index, $\alpha_{\mathrm{mv}}$, is remarkable. Each independent factor of two change at either visual or millimeter wavelengths alters $\alpha_{\mathrm{mv}}$ by about 0.1 . If we assume detection limits of $500 \mathrm{mJy}$ at $1.4 \mathrm{~mm}$ and $1 \mathrm{mJy}$ at $0.7 \mu \mathrm{m}$, then observing only sources bright enough to measure would allow $\alpha_{\mathrm{mv}}$ to range approximately from 0.5 to 1.25 . A proper test of whether the small dispersion we find for $\alpha_{\mathrm{mv}}$ is intrinsic to the sources or due to our small selected sample would require a larger sample of sources. Nevertheless, because different samples (of variable sources) produce the same $\left\langle\alpha_{\mathrm{mv}}\right\rangle$ with small dispersion (e.g., O'Dell et al. 1978; Jones et al. 1981), it seems likely that $\left\langle\alpha_{\mathrm{mv}}\right\rangle$ is of some real physical significance and not entirely a selection effect. Ennis, Neugebauer, and Werner (1982) characterize this region of the spectrum by the ratio of observed $1 \mathrm{~mm}$ flux density to that extrapolated from the IR and find for this quantity a similarly small dispersion among similar sources. Some classes of objects, such as optically selected QSOs, give different results, but $\alpha_{\mathrm{mv}} \approx 0.7$ is very close to the mean spectral index for radio emission from such diverse objects as normal spiral galaxies (Klein and Emerson 1981), Seyfert galaxies (de Bruyn and Wilson 1978), giant radio galaxies (Kellermann, Pauliny-Toth, and Williams 1969), jets of radio galaxies (Bridle 1982), and knots in the jets of quasars (Owen and Puschell 1982). The prevalence of this synchrotron spectral index is commonly believed to be a signature of a widely applicable particle acceleration mechanism. It is possible, therefore, that we are seeing evidence of the same mechanism in action in compact regions of active nuclei as well.

Because the centimeter spectra of these objects (see Fig. 1) are considerably flatter than $\alpha \sim 0.7$, there must 


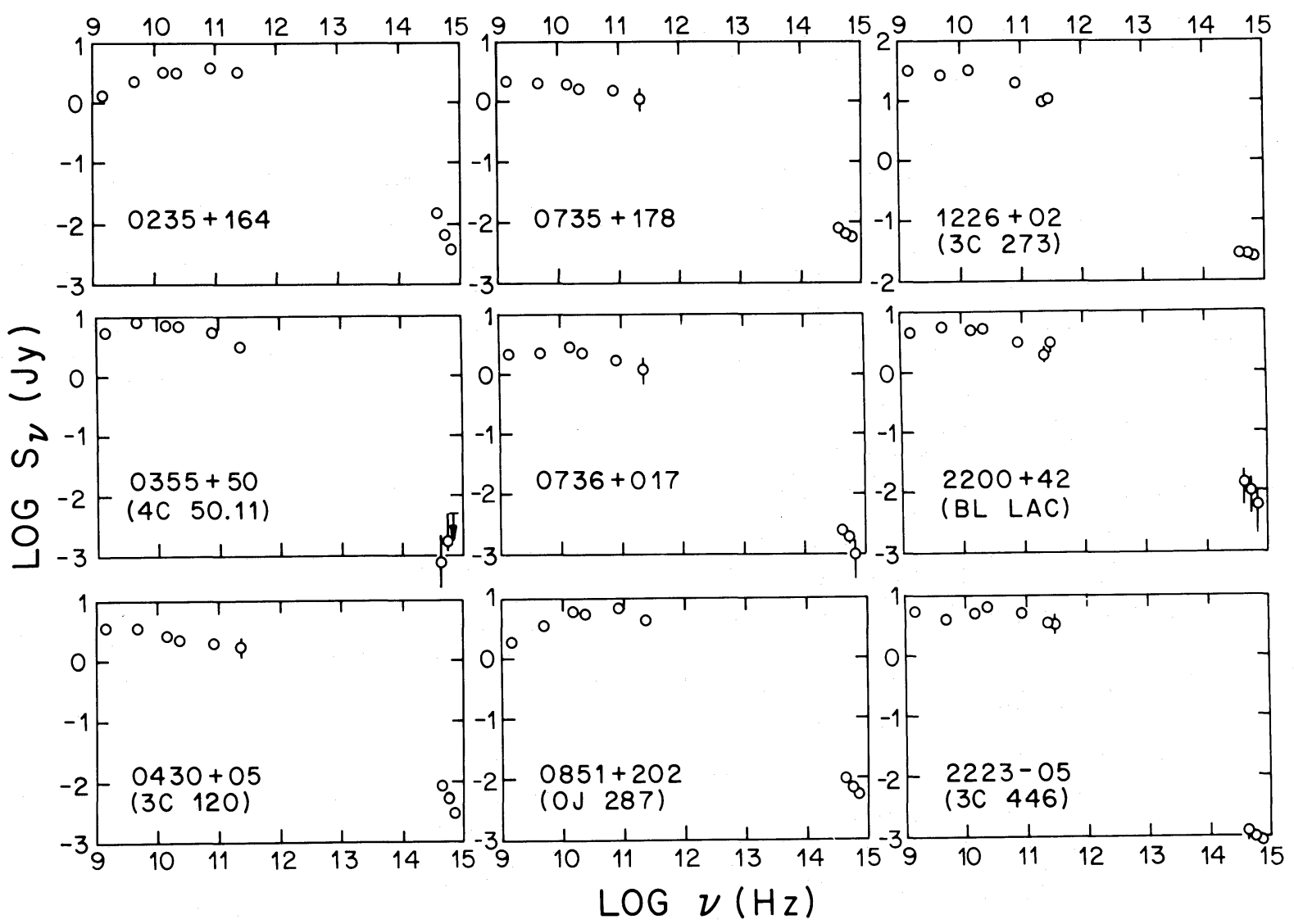

FIG. 1.-Flux density vs. frequency for nine sources measured simultaneously in the radio, the visual, and at $1 \mathrm{~mm}$. The large errors for $0355+50$ and for $2200+42$ in the visual are due to the uncertainty in galactic extinction at these sources' low galactic latitudes. Note the different scale for $3 \mathrm{C} 273$.

be a transition at millimeter or shorter wavelengths. The flatness of the centimeter spectra is usually attributed to the effects of opacity combined with a range of length scales contributing emission at different wavelengths, with the largest scales dominating at the longest wavelengths. The wavelength at which the required steepening toward the infrared begins is then largely determined by the size of the smallest scale emitting regions. In some cases the spectra of Figure 1 begin to steepen between $10^{11} \mathrm{~Hz}(3 \mathrm{~mm})$ and $3 \times 10^{11} \mathrm{~Hz}(1 \mathrm{~mm})$, but, in any case, the transition probably cannot occur at much higher frequencies because of the small scatter in $\alpha_{\mathrm{mv}}$ mentioned above. As discussed by Jones et al. (1981), that leads to a range of about $10^{16}-10^{17} \mathrm{~cm}$ for the smallest size scales.

In contrast to the nearly constant millimeter/visual spectral index, the visual spectral index (see Table 4) varies more or less uniformly over our sample from $0.3 \pm 0.04(3 \mathrm{C} 273)$ to $2.8 \pm 0.3(0235+164)$. This wide variation and the prevalence of indices much larger than 0.7 is shared by the sample of highly polarized quasars (HPQs) of Moore (1981) and the sample of optically violent variables (OVVs) of Sitko et al. (1982), but not shared by a sample of all QSOs or even of all radioselected QSOs. We suggest that the three characteristics of high polarization, violent variability, and large visual spectral index are all manifestations of a "small" emitting region.

If we interpret $\alpha_{\mathrm{mv}} \approx 0.7$ as reflecting the intrinsic energy distribution of accelerated electrons, then over that portion of the spectrum synchrotron and selfCompton radiative losses must be unimportant. Radiative losses steepen the electron energy distribution beyond the injected form. In fact we now show that for electrons radiating at infrared frequencies these energy losses in typical OVVs must be very severe (e.g., Blandford and Rees 1978; Puschell et al. 1983) unless the emitting region is beamed in our direction at nearly the speed of light.

Consider a simple criterion for severe energy losses; namely, whether the electrons can, in free flight, cross the emitting region. Including radiative losses to synchrotron and self-Compton processes, one maximizes the electron lifetimes (roughly) by setting the rates for these two processes equal. Then noting that the observed characteristic synchrotron frequency for an electron of energy $\gamma m c^{2}$, corrected for redshift and bulk motion is $v \sim \gamma^{2} v_{B} \delta /$ $(1+z)$, where $v_{B}$ is the cyclotron frequency, $z$ is the emission line redshift and $\delta=[\gamma(1+\beta \cos \theta)]^{-1}$ is the Doppler shift due to motion relative to the emission line 
reference frame, we can calculate the value of $\delta$ necessary to allow electrons to traverse the emitting region. The principal dependence on $\delta$ comes through the Doppler boost in brightness. The result is

$$
\delta \gtrsim 7\left[\frac{d_{\mathrm{Gpc}}{ }^{3 / 7} S_{\mathrm{mJy}}{ }^{3 / 14}}{r_{16}{ }^{1 / 7} \lambda_{\mu \mathrm{m}}{ }^{5 / 14}}\right](1+z),
$$

where the luminosity distance $d_{\mathrm{Gpc}}$ is in gigaparsecs, the flux density $S$ at wavelength $\lambda_{\mu \mathrm{m}}$ is in millijanskys and the size of the emitting region $r$ is in units of $10^{16} \mathrm{~cm}$. This relation should be true provided the synchrotron spectrum is not much steeper than that expected for the initially injected electron distribution. Using typical parameters appropriate for OVVs we can evaluate the right-hand side in regions where we find spectra not appreciably steeper than $\alpha \approx 0.7$ (from $1 \mathrm{~mm}$ to about $10 \mu \mathrm{m})$. The quantity in square brackets is close to unity, and we are led to the requirement that the emission we are seeing in this region has been boosted by Doppler factors $\delta \sim 10$. This is similar to values determined by other independent considerations such as superluminal motions in radio sources (e.g., Cohen and Unwin 1982) and constraints on X-ray emission (Burbidge, Jones, and O’Dell 1974; Marscher and Broderick 1981). In the visual, where the spectrum is steeper, the inequality of equation (1) need not hold, and energy losses may, in fact, be very large.

The spectrum of $1749+09$ is notable. Table 3 and Figure 2 show the spectrum rising in the radio with $\alpha \approx-0.3$, but, unlike $0235+164$ and $0851+202$ (OJ 287) with similar radio spectral indices, the low $1.4 \mathrm{~mm}$ flux suggests $1749+09$ has a well-defined "spectral break" perhaps indicating the transition to optically thin radiation. In all our other sources the millimeter flux densities lie on more reasonable extrapolations from the radio flux densities and indicate less abrupt transitions to their visual spectra.

\section{b) Variability}

Figure 2 shows the time history of the nine sources for which we have radio and millimeter measurements and that are in common with those for which Jones et al.

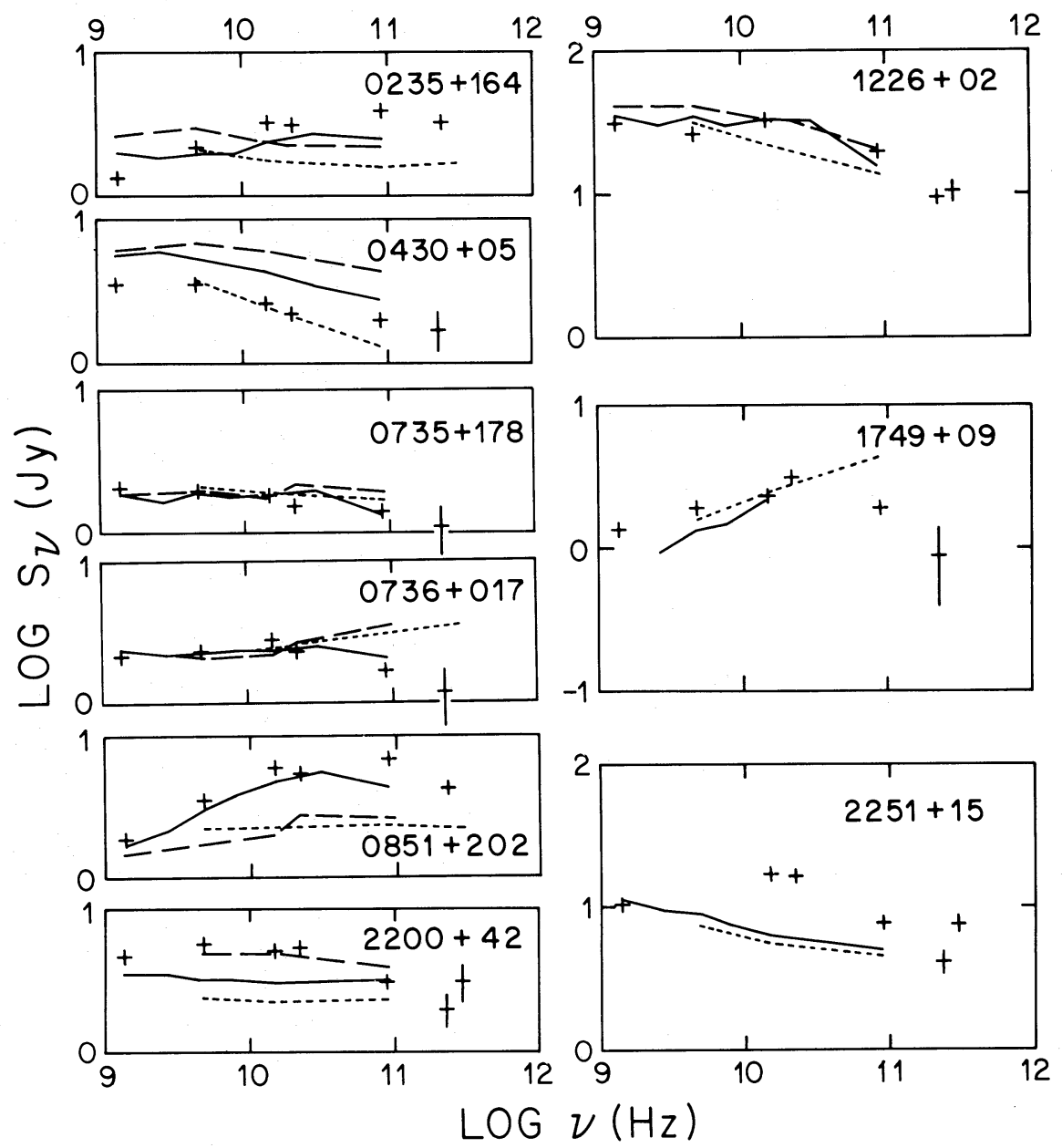

FIG. 2.-Time histories of the radio-millimeter spectra of nine sources. Solid lines are epoch 0 (1977.1); dashed lines are epoch 1 (1978.1); dotted lines are epoch 4 (1979.4); plus signs are the data from this paper: epoch 6 (1981.9). Errors are shown for the $1 \mathrm{~mm}$ points only. 
(1981) reported spectra at previous epochs. Four epochs are shown: epoch 0-1977 January (Owen et al. 1978); epoch 1-1978 January (Owen, Spangler, and Cotton 1980); epoch 4-1979 April/May (Jones et al. 1981 with $3 \mathrm{~mm}$ points for 3C 120 and 3C 273 from Landau, Epstein, and Rather 1980); and epoch 6-1981 December (this paper). (The numbering follows closely that of Jones et al. 1981.)

For most sources the shape of the radio spectrum has changed little over the 5 year period covered although these objects are quite active. The same is true of the visual spectra where comparisons can be made with the data of O'Dell et al. (1978). These facts suggest some reasonably long-term stability to the source structure. It is unlikely, for example, that radio outbursts of random independent homogeneous components would continue to produce spectra of such constant character but different strength.

However Figure 2 shows that the shape of the radio spectra of $2251+15$ and (perhaps) $0235+164$ have changed in recent years. (Changes in the spectral shape of $0235+164$ were noted by O'Dell et al. 1977 and by Balonek and Dent 1980 following its outburst in 1975 October.) Furthermore a comparison with O'Dell et al. (1978) shows that the visual spectral index (between $R$ and $B$ ) of $0736+017$ changed from 0.24 in 1977 March to 1.9 in 1981 December, and that of $0735+178$ changed from 2.95 in 1976 November to 0.82 in 1977 February to 0.77 in 1981 December. In the case of $2251+15$ we are seeing the spectrum just after an outburst, which peaked around 1981.5. It is possible that spectra, such as those shown in Figure 2 for $2200+42$ and $0430+05$, which change in scale but not in shape, result from sampling these active sources at "quiescent" times, whereas the spectrum of an outburst may be peaked in wavelength, modifying the shape of the quiescent spectrum. Changes in spectral shape would be expected if the electrons injected in the outburst suffer radiative losses.

We thank L. Rudnick for extensive discussion; W. Dent, E. Grossman, J. Kenney, W. Kinzel, R. Perley, J. Rodriguez, T. Roellig, and the staff of the NRAO Tucson facility for observing assistance; and Gary Grasdalen and Roger Hildebrand for attempting $10 \mu \mathrm{m}$ and $400 \mu \mathrm{m}$ observations. The work of E. E. E. was supported by U.S. Air Force contract no. F04701-82-C0083. Observations at Palomar were supported by NASA. Work at Mount Lemmon and at the University of Minnesota is supported by NSF grants AST-80-15307 and AST-81-14737.

\section{REFERENCES}

Balonek, T. J. 1982, Ph.D. thesis, University of Massachusetts,

Balonek, T. J., and Dent, W. A. 1980, Ap. J. (Letters), 240, L3.

Blandford, R. D., and Rees, M. J. 1978, in Pittsburgh Conference on BL Lac Objects, ed. A. Wolfe (Pittsburgh: University of Pittsburgh Department of Physics and Astronomy), p. 328.

Bridle, A. H. 1982, in I AU Symposium 97, Extragalactic Radio Sources, ed. D. S. Heeschen and C. M. Wade (Dordrecht: D. Reidel), p. 121

Burbidge, G. R., Jones, T. W., and O'Dell, S. L. 1974, Ap. J., 193, 43 Burstein, D., and Heiles, C. 1982, A.J., 87, 1165

Cohen, M., and Unwin, S. 1982, in IAU Symposium 97, Extragalactic Radio Sources, ed. D. Heeschen and C. Wade (Dordrecht: Reidel), p. 345.

Condon, J. J., O’Dell, S. L., Puschell, J. J., and Stein, W. A. 1981, Ap. J., 246, 624.

de Bruyn, A. G., and Wilson, A. S. 1978, Astr. Ap., 64, 433

Elias, J. H., et al. 1978, Ap. J., 220, 25.

Ennis, D. J., Neugebauer, G., and Werner, M. W. 1982, Ap. J., 262, 460 Epstein, E. E., Landau, R., and Rather, J. D. G. 1980, A.J., 85, 1427.

Hildebrand, R. H., Whitcomb, S. E., Winston, R., Stiening, R. F., Harper, D. A., and Moseley, S. H. 1977, Ap. J., 216, 698.

Jones, T. W., Rudnick, L., Owen, F. N., Puschell, J. J., Ennis, D. J., and Werner, M. W. 1981, Ap. J., 243, 97.

Kellermann, K. I., Pauliny-Toth, I. I., and Williams, P. J. S. 1969 , Ap. J., 157, 1

Kinman, T. D., Wardle, J. F. C., Conklin, E. K., Andrew, B. H., Harvey, G. A., MacLeod, J. M., and Medd, W. J. 1974, A.J., 79, 349. Klein, U., and Emerson, D. T., 1981, Astr. Ap., 94, 29.

Kunkel, W. E., and Rydgren, A. E. 1979, A.J., 84, 633.

Landolt, A. U. 1973, A.J., 78, 959.
Landau, R. 1982, Pub. A.S.P, 94, 600.

Landau, R., Epstein, E. E., and Rather, J. D. G. 1980, A.J., 85, 363.

Marscher, A. P., and Broderick, J. J. 1981, Ap. J. (Letters), 247, L49.

Moore, R. L. 1981, Ph.D. thesis, University of Arizona.

O’Dell, S. L., Puschell, J. J., Stein, W. A., Owen, F. W., Porcas, R. W.,

Mufson, S., Moffett, T. J., and Ulrich, M.-H. 1978, Ap. J., 224, 22.

O’Dell, S. L., Puschell, J. J., Stein, W. A., and Warner, J. W. 1977, Ap. J. (Letters), 214, L105.

Owen, F. N., Porcas, R. W., Mufson, S. L., and Moffett, T. J. 1978, A.J., 83, 685 .

Owen, F. N., and Puschell, J. J. 1982, Ap. J. (Letters), submitted

Owen, F. N., Spangler, S. R., and Cotton, W. D. 1980, A.J., 85, 351.

Puschell, J. J., Jones, T. W., Phillips, A. C., Rudnick, L., Simpson, E.,

Sitko, M. L., Stein, W. A., and Moneti, A. 1983, Ap. J., 265, 625.

Racine, R. 1970, Ap. J. (Letters), 159, L99.

Rieke, G. H., Grasdalen, G. L., Kinman, T. D., Hintzgen, P., Wills,

B. J., and Wills, D. 1976, Nature, 260, 751.

Roellig, T. L. 1980, Ph.D. thesis, Cornell University.

Sandage, A. 1972, Ap. J., 178, 1

Sharov, A. S. 1964, Soviet Astr.-AJ, 7, 689.

Sitko, M. L., Stein, W. A., Zhang, Y.-X., and Wisniewski, W. Z. 1982 , preprint.

Ulich, B. L. 1981, A.J., 86, 1619.

Werner, M. W., Neugebauer, G., Houck, J. R., and Hauser, M. G., 1978, Icarus, 35, 289.

Westphal, J. A., 1974, Infrared Sky Noise Survey, NASA CR 139693, N74-32782 (Springfield, Va.: NTIS).

Whitcomb, S. E., Hildebrand, R. H., Keene, J., Stiening, R. F., and Harper, D. A. 1979, Icarus, 38, 75. 
G. Neugebauer and B. T. Solfer: California Institute of Technology, Physics Department, 320-47, Pasadena, CA 91125

$\lim _{\substack{\infty \\ l \rightarrow 1}}$

J. J. PusChell: Center for Astrophysics and Space Science, University of California, MC C-011, La Jolla, CA 92093

M. W. Werner: NASA Ames Research Center, MS 245-6, Moffett Field, CA 94035 